\title{
A Draw-In Sensor for Process Control and Optimization
}

\author{
Numpon Mahayotsanun, Jian Cao* and Michael Peshkin \\ Department of Mechanical Engineering, Northwestern University, Evanston, IL 60201, USA \\ *Corresponding author: Tel: 847-467-1032, jcao@northwestern.edu
}

\begin{abstract}
Sheet metal forming is one of the major processes in manufacturing and is broadly used due to its high degree of design flexibility and low cost. In the sheet metal forming process, draw-in (planar movement of a sheet periphery) frequently occurs and is one of the most dominated indicators on the success of a forming process. Currently, monitoring and controlling draw-in during each stamping operation requires either time-consuming setup or a significant die modification. Most devices have been used only in laboratory settings. Our goal is to design a draw-in sensor providing high sensitivity in monitoring; ease of setup, measurement and controlling; and eventually be implemented in industry. Our design is based on the mutual inductance principle, which we considered physical factors affecting the characteristics of the draw-in sensor. Two different configurations, single-transducer and double-transducer of our draw-in sensors have been designed and tested. The results showed good linearity, especially for the double-transducer case. The output of the draw-in sensor was affected by the type of sheet metal, dimension of the transducer, and the distance between the transducer and the testing sheet metal. It was found that the result was insensitive to the waviness of the sheet metal if sheet thickness was thin. The invention, implementation, and integration of the draw-in sensor will have an enormous impact on revolutionizing the control of stamping process, will provide solid ground for process variation and uncertainty studies, and ultimately will affect the design decision process.
\end{abstract}

\section{INTRODUCTION}

The sheet metal stamping process is performed by placing a sheet-metal blank over a die cavity and then pushing the metal into the opening with a punch. The blank is held down against the die by a blank holder. As the punch moves further down, the sheet is drawn into the die cavity, which amount depends on the restraining force imposed by a blank holder force and/or draw heads restraining force. Due to the high efficiency of material utilization and the high productivity that stamping enjoys, this process has been widely used in the automotive industry, beverage can industry, appliance and aerospace industry, just to name a few. Various tests and industrial experiences have proven that the amount of draw-in directly affects whether a stamped part will result in wrinkling or tearing, due to excessive or too little draw-in, respectively. Figure 1 illustrates what the draw-in is, which is the amount of blank edge movement. The existing draw-in sensors are mostly made for laboratory use. For example, the LVDT type, which requires significant setup time at industrial setup, becomes too time-consuming or too expensive to use.

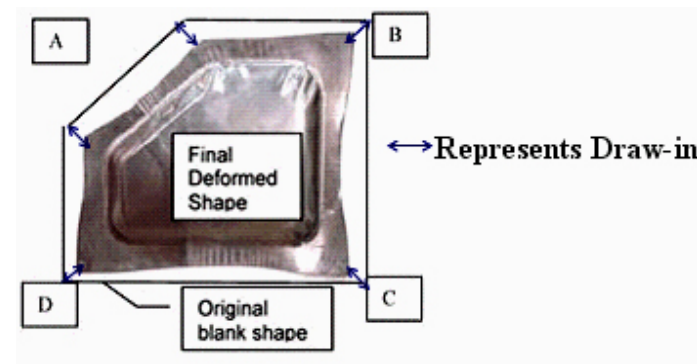

FIGURE 1. The original and final blank shapes in an irregular blank forming. A, B, C, and D show the four corners

With the intention of continuously measuring the drawn-in amount of sheet metals in a stamping process, our approach is to develop a draw-in sensor, using the principle of mutual inductance between two loops as seen in Figure 2 [1, 2]. The excitation current flowing in the primary coil induces Electromotive Force (emf) in the secondary coil. The presence of metal (ferrous or non-ferrous) near the coils affects the magnetic field lines, displayed on the right of Figure 2, changing the degree of mutual inductance. Thus, the induced signals in the secondary coil reflect how much of the coil is covered up. 


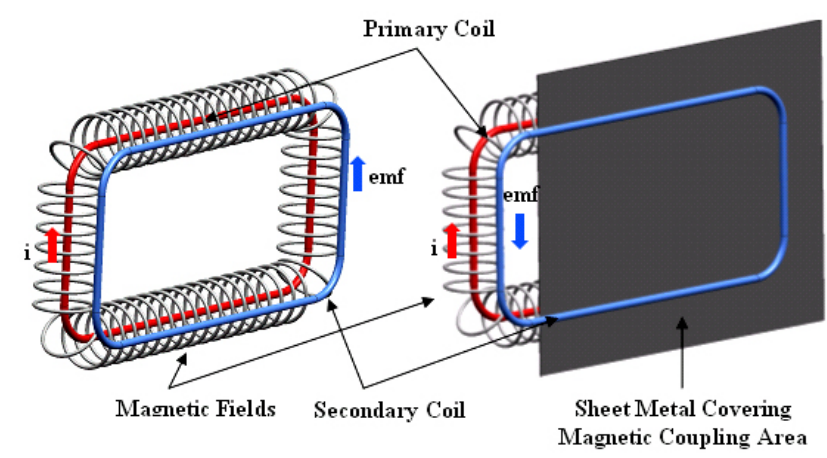

FIGURE 2. A schematic of the sensor element using the mutual inductance used in the double-transducer configuration

Based on the mutual inductance principle, we developed a draw-in sensor by experimenting with a prototype printed on a conventional circuit board to address the need of an affordable and accurate draw-in sensor [3]. We proposed two configurations of the draw-in sensor measurement, which will be further explained in the next two sessions. Measuring results using these two designs will be presented in each of these two sessions, followed by a general discussion on conclusions and future work. Since the ultimate goal is to incorporate the draw-in sensor in the real manufacturing environment, the development of advanced closed-loop control strategies with the drawin sensor will be a future work of this project [4].

\section{SINGLE-TRANSDUCER CONFIGURATION}

Since the sensor cannot interfere with sheet metal during the stamping process, we designed the transducers such that they would be small enough to be embedded in either a die or a blank holder in the stamping tool. Our first design, called singletransducer configuration, was to put together the primary and secondary coils into one transducer as shown in Figures 3 and 4. The principle of mutual inductance between the two loops was still valid, but the movement of the magnetic fields and the disturbance of the sheet metal would differ from what was shown in Figure 2.

Figures $4 \mathrm{a}$ represents the fabricated transducers having a few rounds of outer loops and inner loops. The schematic of the loops is shown in Figure 4b. In order to avoid wear on the sensors and prevent sheet metal from sliding into the transducer slot during the forming process, a thin layer of epoxy was used to cover up the top surface of the transducers as seen in Figure 4c.

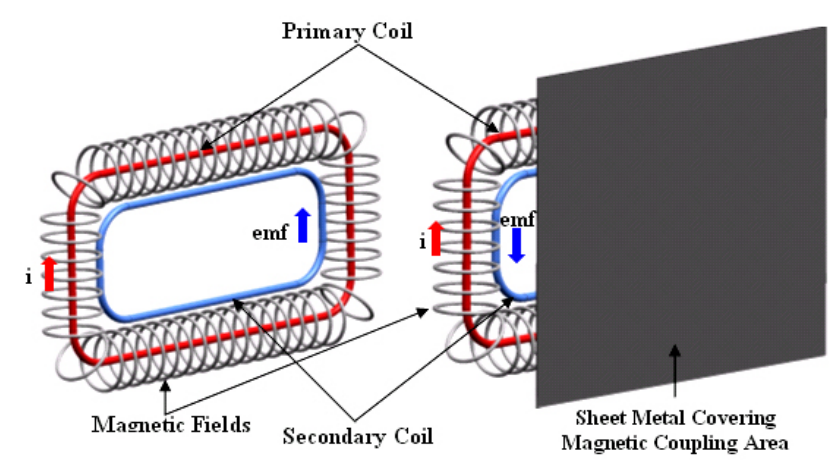

FIGURE 3. A schematic of the single-transducer configuration

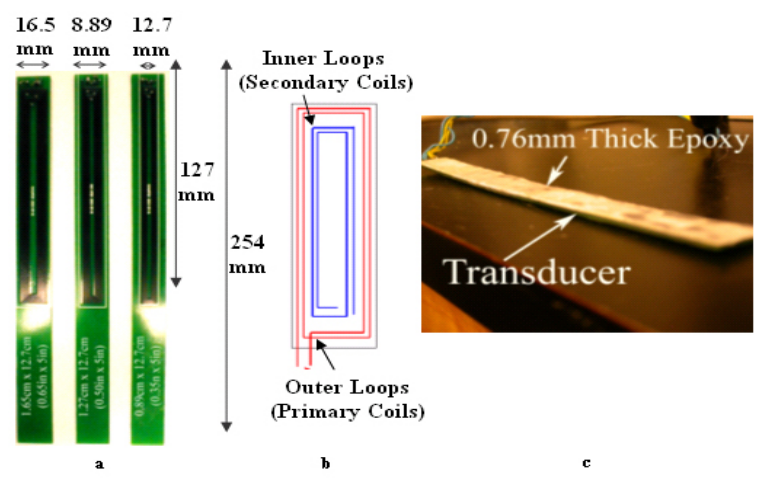

FIGURE 4. a Transducers b. Schematic of the loops in the transducers c. Transducers covered by epoxy

Figure 5 shows the experimental setup of the drawin sensor and the simulated stamping tools were made by steel blocks. In the single-transducer configuration, a transducer covered with epoxy was inserted in the blank holder's slot, which would detect the induced voltage changes caused by the disturbances of the sheet metal located below. The linear position sensor, having $75 \mathrm{~mm}$ detection range, was attached to the sheet metal to determine how much the sheet metal had moved and served as a reference. While the sheet metal was being pulled (manually by using pliers) horizontally to the left, the magnetic coupling area would increase, resulting in the change of induced voltages detected by the transducer. Then, both the transducer and the linear position sensor transmitted signals to the draw-in sensor data acquisition board, which amplified the voltage readings and sent them to the computer for further analysis. Therefore, knowing the amount of voltages indicated by the draw-in sensor, the flow of the sheet metal could be obtained. 


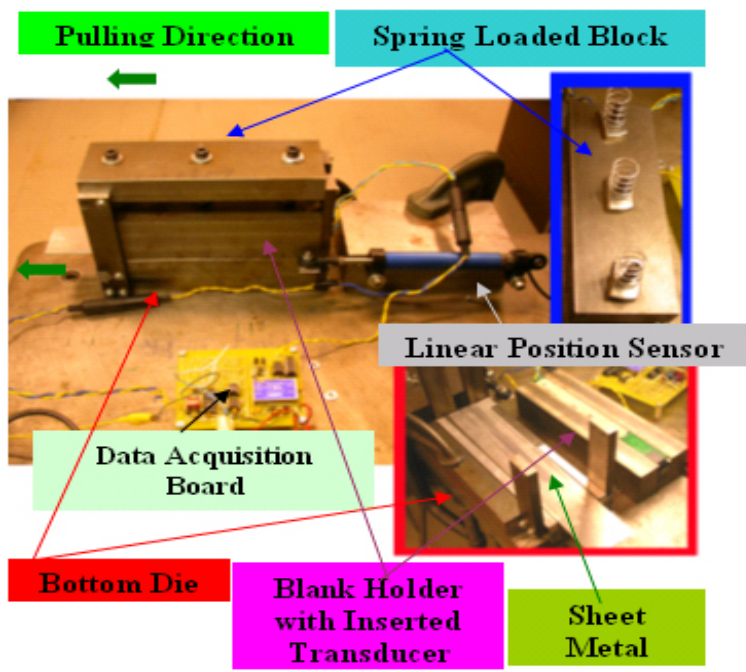

FIGURE 5. Experimental setup

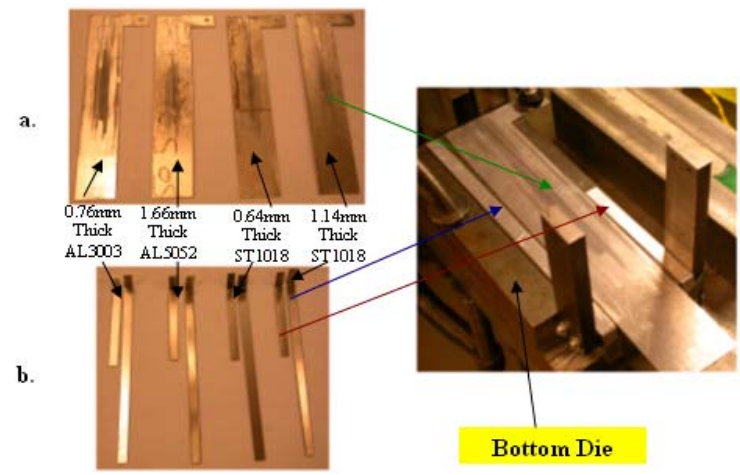

FIGURE 6. a. Flat sheet metals b. Shim sheets to create gaps between transducer and sheet

The experimented sheet metals are displayed in Figure 6a. The hole on the top right of each sheet was for mounting the linear position sensor. The shim sheets, Figure 6b, were used to create gaps between the sheet metal and the transducer, which will be later discussed in the double-transducer configuration.

We considered two major factors influencing the characteristics of the draw-in sensor as presented in Table 1. Specifically, in the remaining of this session, we will illustrate each factor, and why we want to study this factor and the result. The factors are: material, and transducer width. Each combination of different factors was experimented at least five trials in order to show the repeatability of each test as an example shown in Figure 7 . Note that the initial induced voltage was at approximately $6.8 \mathrm{~V}$, indicating that the transducer always detected the mutual inductance in the single-transducer configuration even though the magnetic coupling area was completely covered by the sheet metal.
TABLE 1. Considered parameters in the singletransducer configuration

\begin{tabular}{cc}
\hline Materials & Transducer Widths \\
\hline $0.76 \mathrm{~mm}$ AL3003 & $8.89 \mathrm{~mm}$ \\
$1.66 \mathrm{~mm}$ AL5052 & $12.7 \mathrm{~mm}$ \\
$0.64 \mathrm{~mm}$ ST1018 & $16.5 \mathrm{~mm}$ \\
$1.14 \mathrm{~mm}$ ST1018 & \\
\hline
\end{tabular}

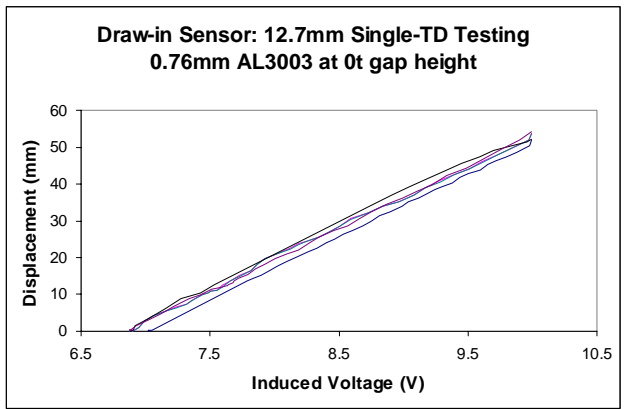

FIGURE 7. Repeatability results of the singletransducer configuration

Material type: We considered four sheet metals to see the effects of material type to the sensitivity of the sensors. The results are illustrated in Figure 8. Note that the slopes of the aluminum sheets were different from those of steel sheets. In the single-transducer configuration, the permeability of sheet metal influenced the magnitude of the mutual inductance. The steel sheets have high permeability, which allow the magnetic fields to penetrate to a certain skip depth, causing high induced voltages while covering the entire magnetic coupling area. On the other hand, aluminum sheets have low permeability, which distort the magnetic fields and disturb the mutual inductance, causing low induced voltage while covering the entire magnetic coupling area. Between the different aluminum sheets, the slopes were slightly different. There existed the slight difference between the slopes of different steel sheets as well.

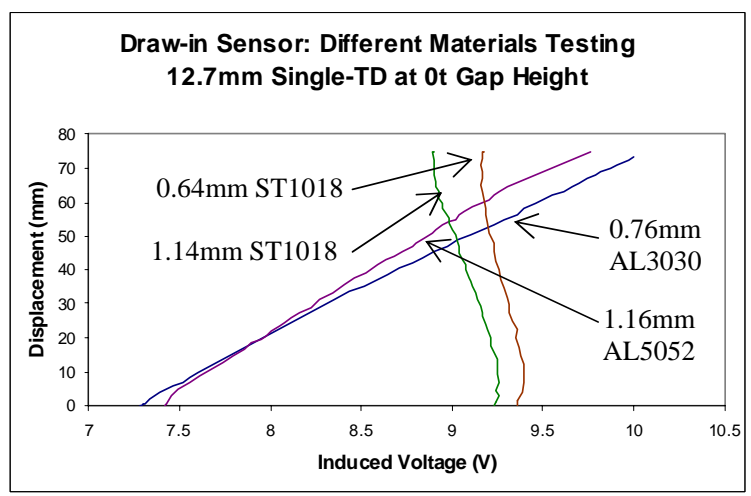

FIGURE 8. Results of different materials in the single-transducer configuration 
Transducer width: Three different transducer widths were considered to see whether the size of the transducers affected the output of the draw-in sensor and the results are presented in Figure 9. It can be observed that wider transducers produced higher induced voltages. The results were as expected since the magnitude of the mutual inductance rises with the increase in the area of the secondary coils.

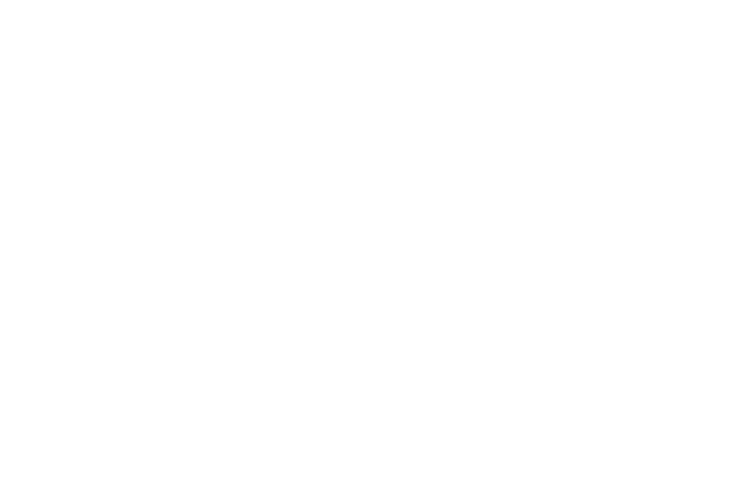

FIGURE 9. Results of different transducer widths in the single-transducer configuration

According to the effect of the transducer width, we could increase the induce voltages by increasing the size of the secondary coils. Therefore, the doubletransducer configuration was introduced and discussed in the next session where the secondary coils were relocated into the outer loops of another transducer.

\section{DOUBLE-TRANSDUCER CONFIGURATION}

The double-transducer configuration also used the mutual inductance principle, which was exactly shown in Figure 2, where the primary and secondary coils were located in separated transducers. Figure 10 shows the locations of each transducer in the experimental setup. In addition to the purpose of increasing the induced voltages, this configuration was aimed to study the effect of the gap distance between the transducer and sheet metal. Also, we could investigate the output of the sensors to wrinkled sheet metals since sheets frequently got wrinkles during the forming process. The effects of wrinkled sheet metals are important since wrinkling can change the forming process by providing more material in one zone and therefore, delaying the tearing. On the other hand, wrinkling presents a difficulty in controlling the springback effectively. Figure 11 shows four wrinkled sheet metals examined in the double-transducer configuration.

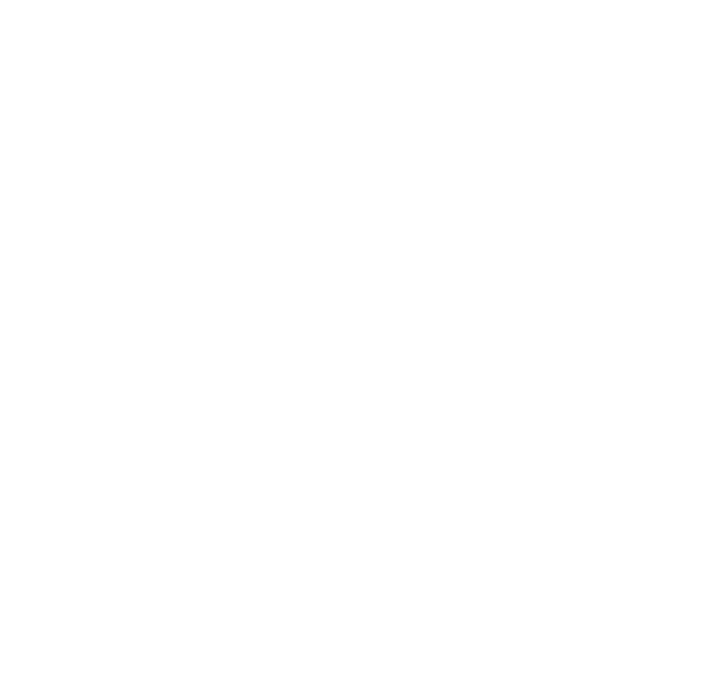

FIGURE 10. Transducers locations in the doubletransducer configuration

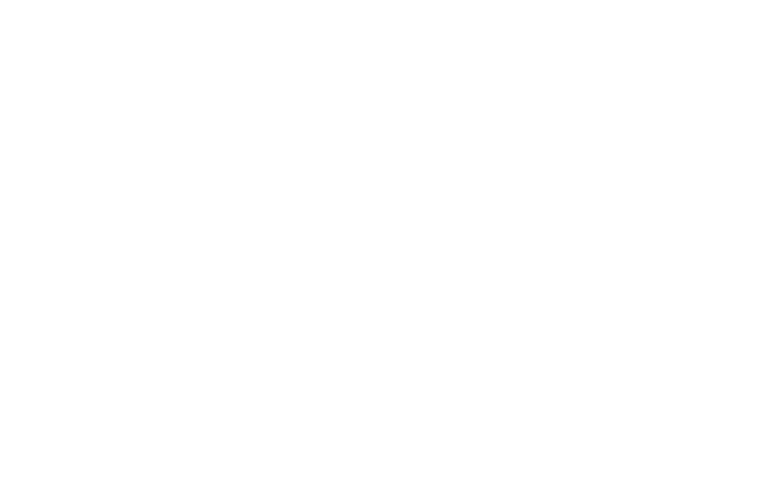

FIGURE 11. Wrinkled sheets

We considered four major factors influencing the characteristics of the draw-in sensor as presented in Table 2. We focused on the following factors: material, transducer width, gap distance between the transducer and sheet metal, and material shape. In the remaining of this session, we will illustrate each factor, why we want to study this factor and the result. Again, each combination of different factors was experimented at least five trials and an example of the repeatability results are shown in Figure 12. Note that this configuration produced better repeatability results compared to those of the single-transducer configuration. In addition, the initial voltages of all the trials were at zero, indicating that the mutual inductance between the two transducers disappeared while being fully covered by the testing sheet metal. 


\begin{tabular}{lccc}
\hline $\begin{array}{l}\text { TABLE 2. Considered parameters in the double-transducer } \\
\text { configuration }\end{array}$ & $\begin{array}{c}\text { Material } \\
\text { Shapes }\end{array}$ & $\begin{array}{c}\text { Transducer } \\
\text { Widths }\end{array}$ & $\begin{array}{c}\text { Gap } \\
\text { Distances } \\
\text { Between } \\
\text { Transducer } \\
\text { and Sheet }\end{array}$ \\
\hline $0.76 \mathrm{~mm}$ & & $8.89 \mathrm{~mm}$ & $0 \mathrm{t}$ \\
AL3003 & Flat & $12.7 \mathrm{~mm}$ & $1 \mathrm{t}$ \\
$1.66 \mathrm{~mm}$ & & & $2 \mathrm{t}$ \\
AL5052 & & $16.5 \mathrm{~mm}$ & $3 \mathrm{t}$ \\
$0.64 \mathrm{~mm}$ & Wrinkled & & \\
ST1018 & & & \\
$1.14 \mathrm{~mm}$ & & & \\
ST1018 & & & \\
\hline
\end{tabular}

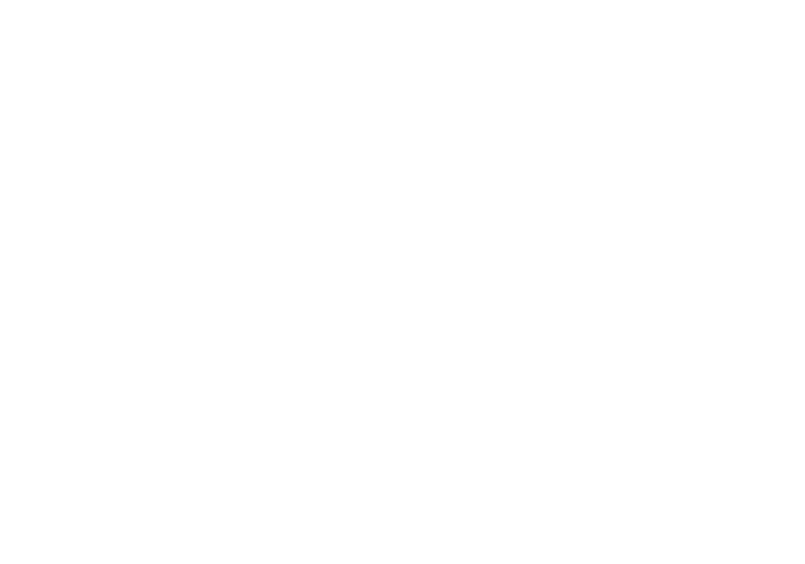

FIGURE 12. Repeatability results of the doubletransducer configuration

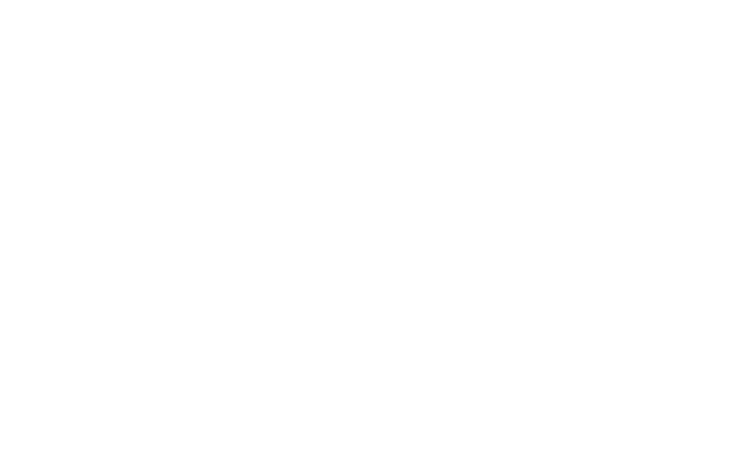

FIGURE 13. Results of different materials in the double-transducer configuration

Material type: We considered four sheet metals to see the effects of material type to the sensitivity of the sensors and the results are shown in Figure 13. As seen in the graph, each material affected the draw-in sensor by providing different slopes on each curve. In contrast with the results of the single-transducer configuration, the double-transducer configuration displayed no differences in the direction of the curves between the aluminum and steel sheets. Since the thickness of each material was many times higher than its skin depth, the magnetic fields could not penetrate through the sheets to the secondary coils. Thus, the induced voltages differed with different sheet metals but their slope sign was the same for all the sheets in the double-transducer configuration.

Transducer width: Three different transducer widths were considered to see whether the size of the transducers affected the output of the draw-in sensor and the results are presented in Figure 14. It can be observed that wider transducers produced higher induced voltages and this behavior also occurred in the single-transducer configuration. Therefore, wider transducers provided higher induced voltages for both configurations.

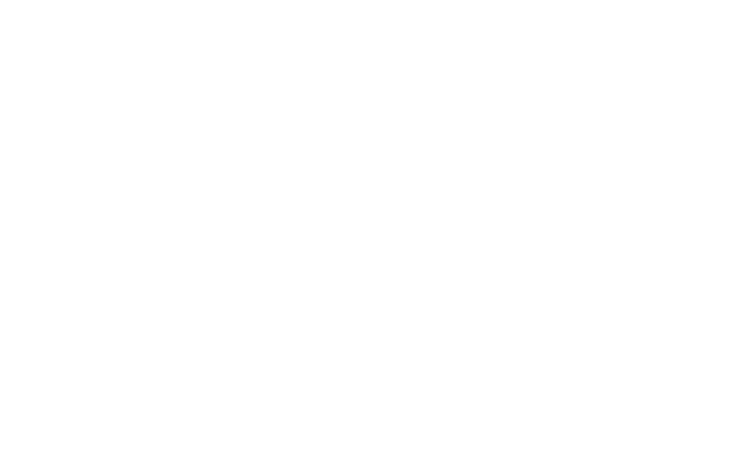

FIGURE 14. Results of different transducer widths in the double-transducer configuration

Gap distance between transducer and sheet metal: To see whether the distance between the transducer and sheet metal affected the sensitivity of the draw-in sensor, four different gap distances between the transducer and sheet metal were considered. The gap distance was increased by adding the shim sheets in between the transducers and the results are shown in Figure 15. Note that $0 \mathrm{t}$ represented "no gap between the transducers", having a long shim sheet and a short shim sheet as shown in Figure 6b. 1t represented "gap distance equals to the thickness of the testing sheet metal", which we added another pair of long and short shim sheets to create a gap. $2 \mathrm{t}$ and $3 \mathrm{t}$ also used the same logic described for 1t. From the graph, it can be seen that higher gap distances created lower induced voltages. 


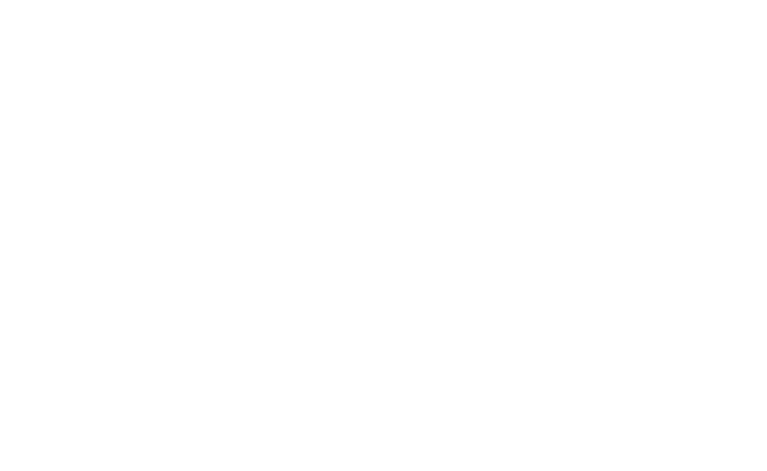

FIGURE 15. Results of different gap heights between transducer and sheet in the double-transducer configuration

Material shape: In order to see the effect of material shape to the output of the draw-in sensor, we considered four wrinkled sheet metals as shown in Figure 11 and compared the results with the flat ones. Figure 16 presents the results of both wrinkled and flat sheet metals. Note that the results of the wrinkled sheets were slightly different than those of the flat sheets for thick materials, $1.66 \mathrm{~mm}$ AL5052 and $1.14 \mathrm{~mm}$ ST1018. However, the draw-in sensor provided similar results for both flat and wrinkled sheets in the case where the sheets were thin, such as $0.76 \mathrm{~mm}$ AL3003 and $0.64 \mathrm{~mm}$ ST1018. As a result, the draw-in sensor is insensitive to material shape when the sheet thickness is approximately smaller than $1 \mathrm{~mm}$.

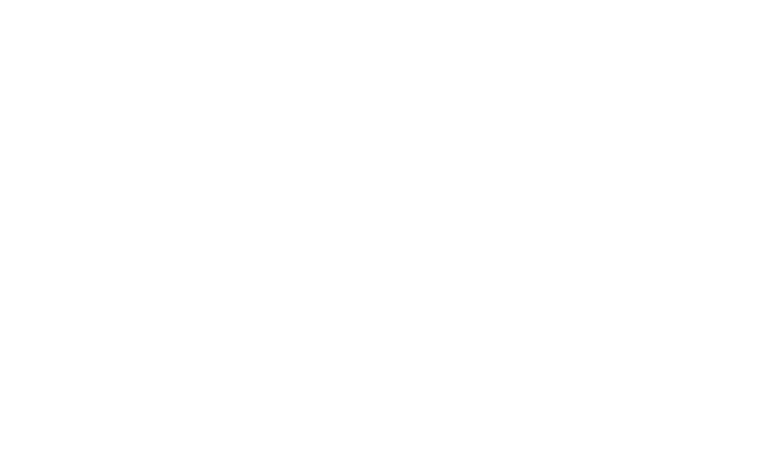

FIGURE 16. Results of different material shapes in the double-transducer configuration

\section{CONCLUSIONS}

The draw-in sensor was developed to measure the flow of materials in the stamping process at a real-time base with no interference with regular stamping press.
Two different configurations, single-transducer and double-transducer, of the draw-in sensor were introduced. The single-transducer configuration produced good linearity. However, the permeability of each material affected the induced voltages such that the initial voltage while the magnetic coupling area was fully covered was not zero. On the other hand, the double-transducer always produces a "zero" readout when the transducers are fully covered. As expected, the sensitivity of the draw-in sensor increased as the width of the transducer increased in both configurations. For the double-transducer configuration, the draw-in sensor provided better repeatability results than those of the single-transducer configuration. Different materials provided different induced voltages but their slope sign was the same for all the sheets of the same type. If the gap distance between the transducer and sheet metal increased, the induced voltages would be reduced. Furthermore, the draw-in sensor was not affected by the shape of the sheet (flat or wrinkle) when the sheet thickness was thinner than $1 \mathrm{~mm}$ in our setup with a gap between the die and the binder being at approximate $3 \mathrm{t}$. This is a very important feature as wrinkling often occurs in the flange area and we wanted our draw-in sensor to be insensitive to the wrinkles.

To further analyze the interaction between these factors and the draw-in sensor, a more sophisticated study such as a DOE study should be performed, for example, to study the effect of wrinkle shape in terms of a normalized gap and its wavelength. Since the ultimate goal is to incorporate the draw-in sensor in the real manufacturing environment, the implementation of draw-in sensor into existing and new tooling will be studied. In addition, the development of advanced closed-loop control strategies with the draw-in sensor will be explored.

\section{REFERENCES}

1. P. Kopczynski, "Lvdts. Theory \& Application". Sensors 1992, 9: 18-22.

2. D-j Choi, C-T Rim; et al., "High Sensitivity Inductive Sensing System for Position Measurement". IEEE Instrumentation and Measurement Technology Conference 2000, 2: 595-599.

3. Cao, J., Lee, J. and Peshkin, M., Northwestern University. Real-time draw-in sensors and methods of fabrication. 2002. U.S. Pat. 6,769,280.

4. N. Krishnan, J. Cao, "Real-time calculation of optimal blank-holder force history in sheet metal forming using an ARMA model". 2002 Japan US Symposium on Flexible Automation, Hiroshima, Japan. 